\title{
Ovarian torsion-A course for severe abdominal pain in children
}

\author{
Maria Kirstine Braendholt Wessel Mantoni, Ulrik Lausten-Thomsen* \\ Department of Pediatrics, Copenhagen University Hospital Holbaek, Holbaek, Denmark \\ Email: *ulau@regionsjaelland.dk
}

Received 14 November 2013; revised 7 December 2013; accepted 15 December 2013

Copyright (C) 2013 Maria Kirstine Braendholt Wessel Mantoni, Ulrik Lausten-Thomsen. This is an open access article distributed under the Creative Commons Attribution License, which permits unrestricted use, distribution, and reproduction in any medium, provided the original work is properly cited.

\begin{abstract}
Ovarian torsion is a rare, albeit important cause of abdominal pain as it may lead to ovarian necrosis if not resolved. Although it has been described in all ages, it is very rare in young children. The primary symptom is abdominal pain, and due to the abundance hereof in a pediatric setting and the significant overlap in the clinical manifestations between ovarian torsion and more common entities such as appendicitis, a high level of suspicion needs to be maintained. We report the case of a young girl with a history of severe abdominal pain. A primary hospitalization at the age of $1 \frac{1 / 2}{2}$ years resolved quickly and spontaneously without any imaging. At the age of 2 years and 10 months, she was once again hospitalized for severe abdominal pain. A CT scan shoved a large dermoid cyst and possible torsion of the ovary. An exploratory laparotomy confirmed the diagnosis and oophorectomy was performed. Histopathological examination of the cyst demonstrated a $5 \times 5 \times 6 \mathrm{~cm}$ mature cystic teratoma without malignancy. This case illustrates how a high level of suspicion for ovarian torsion needs to be maintained, even in otherwise healthy children.
\end{abstract}

Keywords: Childhood Ovarian Torsion; Mature Cystic Teratoma; Dermoid Cyst

\section{INTRODUCTION}

Ovarian torsion is a rare, albeit important cause of abdominal pain as it may lead to ovarian necrosis if not resolved [1]. Although it has been described in all ages, it is very rare in young children [2].

The primary symptom is abdominal pain, and due to the abundance hereof in a pediatric setting and the sig-

"Corresponding author. nificant overlap in the clinical manifestations between ovarian torsion and more common enteties such as appendicitis, a high level of suspicion needs to be maintained [3]. We here present the case of a young girl with a large ovarian tumor and subsequent ovarian torsion.

\section{CASE PRESENTATION}

A 2 years and 10 months old girl was referred urgently by her general practitioner to our pediatric emergency department with a history of severe abdominal pain. The entire family had been sick with influenza-like symptoms for two weeks but the girl had deteriorated for two days with increasing distress, crying and suspicion of abdominal pain. The passing of stools had become sparse and malodorous and a few episodes of vomiting were observed shortly before admission.

The child was otherwise healthy, having been born vaginally at term after an uncomplicated pregnancy, including two routine prenatal ultrasound examinations. A previous hospitalization at $1 \frac{1}{2}$ years of age for abdominal pain had resolved very quickly and spontaneously and the child was discharged whiteout imaging.

On examination, the child was awake but crying weakly with discomfort. She was feverish (38.9 celcius) but otherwise clinically stable. She presented a slightly distended and very tender abdomen with muscular defense hampering detailed clinical evaluation of the abdomen. To distinguish between possible alternative diagnoses, a subsequent low-dose abdominal CT scan without administration of a contrast agent was carried out shortly after. It revealed a $5 \times 5 \times 6 \mathrm{~cm}$ large inhomogeneous cystic tumor and possible torsion of the right ovary. The tumor appeared to contain a large cystic area, as well as fatty tissue and hyperdense calcifications that were interpreted as a possible tooth germ (Figure 1).

The child was referred to the department of pediatric surgery and an exploratory laparotomy was preformed 


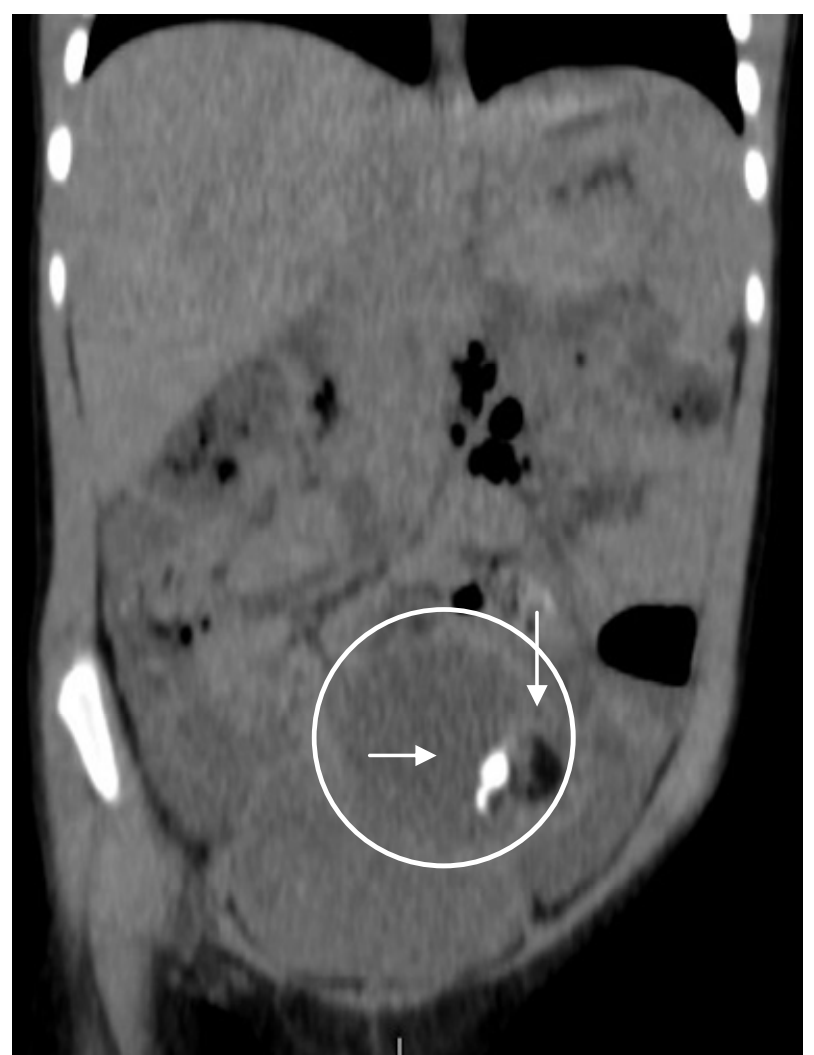

Figure 1. Pre-surgery low-dose pediatric abdominal CT scan revealing a large inhomogeneous cystic process (circle) containing hyperdense calcifications (horizontal arrow), hypodense fatty tissue (vertical arrow), and cystic liquid.

confirming the presence of a large cyst and torsion of the right ovary without any signs of additional pathology.

An ovary preserving surgical intervention was not possible due to both the torsion and the size of the cyst. An uncomplicated oophorectomy was performed leaving the apparently well vascularized and unharmed fallopian tube in situ.

Standard blood samples including HcG and $\alpha$-fetoprotein were normal and subsequent histopathological examination of the cyst confirmed the diagnosis of a mature cystic teratoma measuring $5 \times 5 \times 6 \mathrm{~cm}$ without any indication of malignancy.

The child made an uncomplicated recovery and was discharged after 5 days. At subsequent control after 3 months a standard ultrasound demonstrated a normally looking left ovary. The parents described the child to be even more thriving than before surgery and they hypothesized in retrospect that the child may have been bothered by intermittent discomfort from the cyst in the months and years leading up to surgery.

\section{DISCUSSION}

Ovarian torsion is infrequent and even more so in a pediatric setting as children only account for approximately
$15 \%$ of all cases of ovarian torsion [1]. The exact incidence is unknown, but a large study comprising 1965 cases estimated the annual incidence to be 4.9 per 100.000 females aged 1 to 20 years [2]. Ovarian torsion is overall more common after menarche and only $2 \%$ of cases occur before 5 years, resulting in an annual incidence of 9.8 per 10 million girls aged 1 to 5 [2].

\subsection{Patophysiological Mechanism}

The patophysiological mechanism causing this condition is the twisting of the ovary on its pedicle which hinders blood and lymphatic flow. As a consequence, the ovary becomes engorged and edematous which leads to increasing parenchymal pressure and ultimately may lead to arterial occlusion and ovarian infarction [2]. The twisting may involve the ovary, the fallopian tube, or both but as the symptoms are clinically indistinguishable this can only be determinated perioperatively [4].

The condition classically presents with sudden onset of severe, constant, unilateral pain in the pelvis or lower abdomen. Notably, the pain may be episodic from spontaneous torsion and detorsion [3].

This is illustrated in the presented case as the previous episode of hospitalization for severe abdominal pain at the age of $1 \frac{1}{2}$ may very well represent an episode of torsion and detorsion in this child. At that time, no surgical intervention or imaging was deemed necessary, as the pain subsided quickly and spontaneously and therefore there is no way of knowing for sure. It is however well described that many patients with ovarian torsion can recall episodes of similar pain in the past $[4,5]$.

Furthermore, this is also in concordance with the parents' retrospective impression of episodes of abdominal discomfort although the latter also can be attributed to recall bias.

\subsection{Mature Cystic Teratoma}

Mature cystic teratoma (often called dermoid cyst) is a germ cell tumor composed of well-differentiated tissues derived from the three germ cell layers: endoderm, mesoderm and ectoderm. Germ cell tumors are the most common ovarian neoplasms comprising 20\% - 25\% of all ovarian neoplasms with a peak incidence between ages 20 and 40, and over 95\% of these neoplasms are benign mature teratomas or dermoid cysts [6]. However, in the first two decades of life up to one-third are malignant [6]. The recommended management of dermoid cyst is generally surgical excision due to the risk of ovarian torsion, spontaneous rupture, and risk of malignancy.

Traditionally, dermoid cysts were removed via laparotomy, but current trend is towards minimal surgical 
techniques using laparoscopic excision [7].

\section{CONCLUSION}

In the presented case, ovary preserving surgical intervention was not possible due to ischemic injury following the torsion. Although speculative, it is not impossible that ovary preserving surgical intervention may have been an option at an earlier stage and consequently the case illustrates the need for maintaining a high level of suspicion for conditions requiring surgical intervention, even in otherwise healthy children.

\section{REFERENCES}

[1] Hibbard, L.T. (1985) Adnexal torsion. American Journal of Obstetrics and Gynecology, 152, 456-461. http://dx.doi.org/10.1016/S0002-9378(85)80157-5

[2] Guthrie, B.D., Adler, M.D. and Powell, E.C. (2010) Incidence and trends of pediatric ovarian torsion hospitalizations in the United States, 2000-2006. Pediatrics, 125, 532-538. http://dx.doi.org/10.1542/peds.2009-1360

[3] Schmitt, E.R. and Ngai, S.S. (2013) Twist and shout!
Pediatric ovarien torsion clinical updata and case discussion. Pediatric Emergency Care, 29, 518-523. http://dx.doi.org/10.1097/PEC.0b013e31828a7822

[4] Rossi, B.V., Ference, E.H., Zurakowski, D., Scholz, S., Feins, N.R., Chow, J.S. and Laufer, M.R. (2012) The clinical presentation and surgical management of adnexal torsion in the pediatric and adolescent population. Journal of Pediatric and Adolescent Gynecology, 25, 109113. http://dx.doi.org/10.1016/j.jpag.2011.10.006

[5] Celik, A., Ergün, O., Aldemir, H., Ozcan, C., Ozok, G., Erdener, A. and Balýk, E. (2005) Long-term results of conservative management of adnexal torsion in children. Journal of Pediatric Surgery, 40, 704-708. http://dx.doi.org/10.1016/j.jpedsurg.2005.01.008

[6] Berek, J.A. and Natarajan, S. (2007) Ovarian and fallopian tube cancer. In: Berek, J.S., Ed., Berek \& Novak's Gynecology, Lippincott Williams \& Wilkins, Philadelphia, 1457-1547.

[7] Savasi, I., Lacy, J.A., Gerstle, J.T., Stephens, D., Kives, S. and Allan, L. (2009) Management of ovarian dermoid cyst in the pediatric and adolescent population. Journal of Pediatric and Adolescent Gynecology, 22, 360-364. http://dx.doi.org/10.1016/j.jpag.2008.12.008 